\title{
ORIGINAL
}

\section{SÍNTOMAS RESPIRATORIOS Y FUNCIÓN PULMONAR EN NIÑOS DE 6 A 14 AÑOS DE EDAD Y SU RELACIÓN CON LA CONTAMINACIÓN POR MATERIAL PARTICULADO PM $_{10}$ EN SANTA MARTA- COLOMBIA (*)}

\section{Lídice Álvarez Miño (1) y Alexander Salazar Ceballos (2).}

(1) Universidad del Magdalena. Colombia

(2) Universidad del Magdalena. Colombia

(*) Los resultados que presenta este artículo son parte del proyecto Efectos sobre la salud por la contaminación del aire proveniente del sector portuario carbonífero (Drummond, Carbosan y prodeco de Santa Marta) realizado en convenio entre el Ministerio de Ambiente, Vivienda y Desarrollo Territorial y la Universidad del Magdalena.

\begin{abstract}
RESUMEN
Fundamentos: Los efectos del material particulado $\left(\mathrm{PM}_{10}\right)$ sobre la salud respiratoria son de interés en salud pública por el alto riesgo de enfermar y morir de la población expuesta, más aun cuando se trata de niños. El objetivo del estudio fue identificar la asociación entre las concentraciones de $\mathrm{PM}_{10}$ con la presencia de síntomas respiratorios y la alteración de la función pulmonar en niños de 6 a 14 años en la ciudad de Santa Marta.

Método: Estudio transversal. La muestra fue de 305 niños seleccionados aleatoriamente entre los escolares matriculados en los colegios de las zonas identificadas. Se aplicaron dos cuestionarios: (i) la encuesta del International Study of Asthma Allergies in Childhood (ISAAC), para identificar síntomas de rinitis alérgica y asma y (ii) la encuesta de identificación de co-variables en el ambiente domiciliario. Se realizaron espirometrías para evaluar la función pulmonar. Para caracterizar la exposición se definieron cuatro zonas de la ciudad en las que se ubicaron muestreadores de bajo volumen fmrOMNI, para medir las concentraciones de $\mathrm{PM}_{10}$. Se utilizó EpiInfo 3.5.3 para los análisis bivariados $\left(\mathrm{chi}^{2}\right)$ y multivariados (regresión logística) y se calcularon odds ratio (OR).

Resultados: La prevalencia de síntomas respiratorios en la población estudiada fue de $39,3 \%$. Vivir en zonas expuestas $(\mathrm{ORa}=2,19 \mathrm{p}=0,0015)$ y tener gatos $(\mathrm{ORa}=1,79 \mathrm{p}=0,0389)$ aumentaron el riesgo de presentar síntomas del tracto respiratorio superior.

Conclusiones: En escolares de 6 a 14 años la exposición a concentraciones de $\mathrm{PM}_{10}$ superiores a $70 \mu \mathrm{g} / \mathrm{m} 3$ aumenta el riesgo de presentar síntomas de tracto respiratorio superior y de alterar la función pulmonar.

Palabras clave: Material particulado. Enfermedades respiratorias. Espirometría. Contaminantes del aire. Salud del niño.
\end{abstract}

Correspondencia

Lídice Álvarez Miño

Universidad del Magdalena.

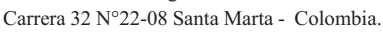

lidice@lycos.com y/o lalvarezm@unimagdalena.edu.co

\section{ABSTRACT \\ Respiratory Symptoms and Lung Function in Children Aged 6-14 Years and Their Relationship with Particulate Matter $\mathbf{P M}_{10}$ in Santa Marta, Colombia}

Background: The effects of particulate matter $\left(\mathrm{PM}_{10}\right)$ on respiratory health are a public health interest because of the high risk of disease and death in the exposed population, more so when it comes to children. The goal was to identify the relationship between concentrations of $\mathrm{PM}_{10}$ and presence of respiratory symptoms and impaired lung function in children aged 6-14 years in the city of Santa Marta..

Method: A cross sectional study was developed. The sample consisted of 305 randomly selected school children enrolled in schools in the areas. Two surveys were carried out: (i) the survey of the International Study of Asthma Allergies in Childhood (ISAAC), to identify symptoms of allergic rhinitis and asthma, and (ii) the survey to identify co-variates in the home environment. Spirometry was performed to assess lung function. To characterize exposure defined four areas of the city where they were located fmrOMNI low volume samplers to measure $\mathrm{PM}_{10}$ concentrations. EpiInfo 3.5.3 was used for bivariate analysis (Chi2) and multivariate (logistic regression) and epidemiological measures (OR).

Results: The prevalence of respiratory symptoms in the study population was $39.3 \%$. Living in exposed areas increases the risk of suffer respiratory symptoms in upper tract $(\mathrm{ORa}=2,19 \mathrm{p}=0,0015)$ and having cats (ORa $=1,79 \mathrm{p}=0,0389$ ).

Conclusions: In children aged 6-14 years exposed to PM10 concentrations higher than $70 \mu \mathrm{g} / \mathrm{m} 3$ the risk of upper respiratory tract symptoms increases and lung function is altered.

Keyword: Particulate matter. Respiratory tract diseases. Spirometry. Air pollution. Child. 


\section{INTRODUCCIÓN}

La relación entre el material particulado y sus efectos sobre la salud respiratoria es uno de los principales intereses de la salud pública por el alto riesgo que producen en la población expuesta de enfermar y morir. El efecto de los contaminantes en la mortalidad es uno de los más estudiados ${ }^{1,2}$. Existe evidencia de que la contaminación del aire está asociada con enfermedad respiratoria, en especial en niños menores de 14 $a_{n}{ }^{3}{ }^{3}$. Los niños que viven en áreas contaminadas buscan más atención médica por síntomas respiratorios y crisis de asma ${ }^{4,5}$, lo cual constituye un problema por los costos de atención en salud ${ }^{6}$, tanto por parte del sistema como de las familias, y afecta directamente a los niños que no pueden realizar sus actividades cotidianas?

En un estudio realizado en Río de Janeiro se encontró asociación entre la exposición a $\mathrm{PM}_{10}$ y $\mathrm{NO}_{2}$ incluso dentro de los niveles aceptables de contaminación atmosférica, con una disminución en la función pulmonar en niños ${ }^{8}$. En Chiguagua, México, se evidenció cómo un incremento de $20 \mathrm{mg} / \mathrm{m}^{3}$ en la concentración de $\mathrm{PM}_{10}$ se relaciona con un incremento del $4,97 \%$ (IC 95\%, 0,97-9,13) en las consultas por asma con un retraso de cinco días. En Temuco, Chile, y La Habana, Cuba, se identificó que existe relación entre la contaminación atmosférica y la enfermedad respiratoria aguda, para establecer procesos de alerta ${ }^{10,11}$. En Bogotá, Colombia, se estableció una asociación significativa entre la enfermedad respiratoria aguda en niños y el $\mathrm{PM}_{10}{ }^{12}$. Para la misma ciudad, en un estudio de cohorte prospectivo ${ }^{13}$ con población de niños menores de 14 años, resultó que el número total de consultas por enfermedad respiratoria de esta población está asociado con la concentración de $\mathrm{PM}_{10}$ en los días precedentes.

Otras investigaciones han encontrado asociación positiva entre la mortalidad por eventos cardiorespiratorios y enfermedad pulmonar obstructiva con la variación temporal de la concentración de $\mathrm{PM}_{10} \mathrm{y}$ $\mathrm{PM}_{2.5}{ }^{14-16}$.

En Santa Marta, Colombia, se encuentran estudios de caracterización de la contaminación que identificaron concentraciones de material particulado en niveles superiores a las establecidas en la normatividad ${ }^{17,18}$. Y un estudio descriptivo sobre el asma ${ }^{19}$ que identificó alérgenos intradomiciliarios y presencia de sintomatología de asma en niños.

El objetivo de este estudio fue identificar la relación entre la concentración $\mathrm{PM}_{10}$ con la presencia de síntomas respiratorios y alteración de la función pulmonar para un grupo de niños y niñas de 6 a 14 años en 4 zonas con diferentes niveles de contaminación de la ciudad de Santa Marta, Colombia.

\section{SUJETOS Y MÉTODOS}

Se desarrolló un estudio transversal en 2009. Para caracterizar la exposición a material particulado se definieron cuatro zonas que, siguiendo las recomendaciones de la $\mathrm{OMS}^{20}$, se clasificaron en: expuestas, aquellas que tenían una concentración superior a $70 \mu \mathrm{g} / \mathrm{m}^{3}$ (Gaira y Pescaito) y no expuestas (Don Jaca-Cristo Rey y Mamatoco). Las mediciones de $\mathrm{PM}_{10}$ las realizaron especialistas con equipos de bajo volumen fmrOMNI y se contó con el apoyo de los datos compilados por la Corporación Regional Autónoma del Magdalena-CORPAMAG.

Por muestreo aleatorio (IC95\%) en los colegios de las zonas seleccionadas, se seleccionó a 305 niños. Los criterios de inclusión fueron: edad entre 6 y 14 años, haber vivido durante los dos últimos años en las zonas seleccionadas, estudiar en las escuelas ubicadas en dichas zonas y que los padres firmaran el consentimiento informado. 
Las variables dependientes medidas fueron la presencia de síntomas respiratorios y la alteración de la función pulmonar. Los síntomas se identificaron a través de una encuesta adaptada del International Study of Asthma Allergies in Childhood (ISAAC) $)^{21,22}$ y se agruparon en: (i) síntomas del tracto respiratorio superior (TRS): congestión nasal, prurito, estornudo, rinorrea y epifora; (ii) síntomas del tracto respiratorio inferior (TRI): despertarse por tos, dificultad respiratoria con el ejercicio y dificultad al hablar. Para medir la alteración de la función pulmonar se realizaron espirometrías, siguiendo las especificaciones de la Sociedad Torácica Americana ${ }^{23}$ y la Sociedad RespiratoriaEuropea ${ }^{24}$ con espirómetros portables marca Discovery-2. Todas las espirometrías fueron realizadas por profesionales con experiencia en el procedimiento (enfermeras profesionales con terapeuta respiratorio), teniendo en cuenta los criterios de confiabilidad y reproducibilidad a través de la aplicación de un protocolo específico. La lectura e interpretación de los resultados la realizó inicialmente una terapeuta respiratoria y después un neumólogo a través de parámetros espirométricos que posteriormente permitieron establecer los patrones espirométricos (tabla 1). A este procedimiento faltaron 16 niños.

Las variables independientes consideradas fueron la concentración de $\mathrm{PM}^{10} \mathrm{y}$ las covariables o contaminantes del ambiente domiciliario, las cuales se identificaron a través de una encuesta aplicada en las viviendas, siguiendo la metodología de un estudio realizado en Bucaramanga, Colom$\mathrm{bia}^{25}$. Estas covariables incluyeron aspectos como: consumo de tabaco en la vivienda, contaminantes de la cocina, presencia de animales domésticos en la vivienda (perros, gatos, aves, etc.), condiciones de la infraestructura de la vivienda (material de techos, paredes, pisos, etc.), contaminantes alrededor de la vivienda (ej. basureros, flujo vehicular, fábricas, etc.) y antecedentes patológicos familiares (gripas, asma, bronquitis, etc.)

Para el análisis de la información, inicialmente, se utilizó la prueba de $\mathrm{chi}^{2}$ con el fin de identificar la relación (i) entre presencia de síntomas respiratorios totales, estratificados por sexo y edad, con la zona de exposición y (ii) entre alteración de la función pulmonar con la zona de exposición. Posteriormente, se midió la asociación estadística a través de Odds Ratio (OR) utilizando dos modelos. El primero permitió hallar la asociación entre la presencia de síntomas (en total y agrupados en TRS y TRI) con la exposición a $\mathrm{PM}_{10}$ y con las covariables. El segundo modelo de análisis se utilizó para identificar asociación estadística entre la alteración de la función pulmonar con la exposición a $\mathrm{PM}_{10} \mathrm{y}$ covariables. Inicial-

Tabla 1

\section{Clasificación de los patrones espirométricos}

\begin{tabular}{|l|c|c|c|}
\hline \multicolumn{1}{|c|}{ Obstrucción pura } & Proceso restrictivo & Alteración mixta & Normal \\
\hline $\begin{array}{l}\text { FVC normal. } \\
\text { FEV1 disminuido }\end{array}$ & FVC disminuido. & FVC disminuido. & FVC normal $(\geq 80 \%)$ \\
FEV1/FVC disminuido & FEV1/FVC normal & FEV1/FVC disminuido & FEV1 normal $(\geq 80 \%)$ \\
FEV1/FVC normal $(\geq 75 \%)$
\end{tabular}

Parámetros: FVC: capacidad vital forzada;

FEV 1: volumen máximo espirado en el primer segundo de una espiración forzada; FEV1/FVC: porcentaje del volumen total espirado en el primer segundo

Fuente: Elaboración propia con base en. De Lucas P, Marañón G. Patrones diagnósticos en la espirometrías. Madrid. Disponible en: http://www.med.uva.es/ biofis/fisio/Respiratorio/Resp2012/patrones_diagnosticos_espirometria.pdf 
mente se midió la asociación entre cada parámetro espirométrico disminuido por zona de exposición y posteriormente los patrones espirométricos se dicotomizaron en normal y anormal (alteración mixta, obstrucción pura y proceso restrictivo) para poder establecer la asociación entre la función pulmonar con la exposición a $\mathrm{PM}_{10} \mathrm{y}$ covariables.

Finalmente, el análisis multivariado se realizó a través de regresión logística no condicionada con las variables que fueron identificadas como factor de riesgo con significancia estadística ( $\mathrm{p}<0,05)$, por lo cual, se consideraron como variables independientes únicamente las zonas de exposición y la presencia de gatos en la casa. Para el análisis se utilizó el programa EpiInfo 3.5.3.

\section{RESULTADOS}

Se definieron como zonas no expuestas Don Jaca-Cristo Rey (DJCR) y Mamatoco y como zonas expuestas Gaira y Pescaíto, las cuales presentaron más de $70 \mu \mathrm{g} / \mathrm{m}^{3}$ de $\mathrm{PM}_{10}$ (tabla 2).

La prevalencia total de síntomas respiratorios en la población estudiada fue de $39,3 \%$. La prevalencia en las zonas expuestas $(45,4 \%)$ fue mayor que la de las zonas no expuestas $(34,1 \%)$. Se observó una relación estadísticamente significativa $\left(\mathrm{chi}^{2}=4\right.$ y $\left.\mathrm{p}=0,04\right)$ entre vivir en zona expuesta y la presencia de síntomas respiratorios (tabla 3 ).
Según el modelo de asociación estadística entre la presencia de síntomas respiratorios totales con la exposición a $\mathrm{PM}_{10}$, se encontró que los niños que habitaban en zonas expuestas tuvieron mayor probabilidad de presentar síntomas respiratorios con un $\mathrm{OR}=1,6$ (IC95\% 1,009-2,614). Además, se observó asociación estadísticamente significativa entre tener síntomas del TRS con vivir en zonas expuestas OR=2,3 (IC95\%, $1,4-3,7)$ y con la tenencia de gatos $\mathrm{OR}=1,9$ (IC95\%, 1,1-3,2). Se encontró una prevalencia de síntomas del TRS del 34,8\% en los niños de zonas expuestas (tabla 4). La prevalencia de síntomas del TRI fue de $11,4 \%$ y no se encontró asociación estadísticamente significativa entre vivir en zona expuesta y presentar síntomas en TRI.

El segundo modelo de análisis, que consistió en la realización de pruebas de OR para identificar asociación entre la alteración en la función pulmonar con la exposición a $\mathrm{PM}_{10}$ y covariables, permitió hallar asociación estadísticamente significativa entre la disminución de los parámetros espirométricos y vivir en zona expuesta a $\mathrm{PM}_{10}$ (tabla 5). Además, se observó asociación epidemiológica entre presentar alteración de la función pulmonar y vivir en una zona expuesta con un $\mathrm{OR}=2,97$ (IC 95\% 1,844,79 ) (tabla 6). Con relación a las covariables no se encontró asociación estadísticamente significativa con la alteración de la función pulmonar.

Por regresión logística se confirmó que vivir en zona expuesta [OR ajustado $=2,19$

Tabla 2

Medias de concentración de $\mathrm{PM}_{10}$ para cada zona expuesta y no expuesta

\begin{tabular}{|c|c|c|c|c|}
\hline \multirow{2}{*}{$\begin{array}{c}\text { PM }_{10} \\
\boldsymbol{\mu} \mathbf{g} / \mathbf{m} 3)\end{array}$} & \multicolumn{2}{|c|}{ Zonas no expuestas } & \multicolumn{2}{c|}{ Zonas expuestas } \\
\cline { 2 - 5 } & $\begin{array}{c}\text { Don Jaca-Cristo Rey } \\
\text { (DJCR) }\end{array}$ & Mamatoco & Gaira & Pescaíto \\
\hline $\begin{array}{c}\text { Media de la concentración } \\
\text { (IC 95\%) }\end{array}$ & $\begin{array}{c}39,24 \\
\text { (IC: } 36,84-41,63)\end{array}$ & $\begin{array}{c}58,5 \\
\text { (IC: } 50,4-66,6)\end{array}$ & $\begin{array}{c}83,72 \\
\text { (IC: } 67,9-99,5)\end{array}$ & $\begin{array}{c}74,73 \\
\text { (IC: } 59,4-90,03)\end{array}$ \\
\hline
\end{tabular}


Tabla 3

Relación entre presencia de síntomas respiratorios con sexo, edad, zona de exposición y covariables

\begin{tabular}{|c|c|c|c|c|c|c|c|c|c|}
\hline & \multirow[t]{2}{*}{ Variables } & \multicolumn{2}{|c|}{$\begin{array}{c}\text { Con } \\
\text { síntomas }\end{array}$} & \multicolumn{2}{|c|}{$\begin{array}{c}\text { Sin } \\
\text { síntomas }\end{array}$} & \multirow[t]{2}{*}{ Total } & \multirow[t]{2}{*}{$\operatorname{chi}^{2}$} & \multirow[t]{2}{*}{$\mathrm{p}$} & \multirow{2}{*}{$\begin{array}{c}\text { OR } \\
\mathrm{IC} 95 \%\end{array}$} \\
\hline & & $\mathrm{n}$ & $(\%)$ & $\mathrm{n}$ & $(\%)$ & & & & \\
\hline \multirow{2}{*}{ Sexo } & Femenino & 71 & 39,7 & 108 & 60,3 & 179 & \multirow{2}{*}{0,02} & \multirow{2}{*}{0,89} & \multirow{2}{*}{ - } \\
\hline & Masculino & 49 & 38,9 & 77 & 61,1 & 126 & & & \\
\hline \multirow{2}{*}{ Edad } & $<9$ & 54 & 46,2 & 63 & 53,8 & 117 & \multirow{2}{*}{3,24} & \multirow{2}{*}{0,07} & \multirow{2}{*}{-} \\
\hline & $>9$ & 66 & 35,1 & 122 & 64,9 & 188 & & & \\
\hline \multirow{2}{*}{ Zona } & $\begin{array}{c}\text { Expuesta } \\
\text { (Pescaito-Gaira) } \\
\mathrm{PM}_{10}>\text { de } 70 \mu \mathrm{g} / \mathrm{m} 3\end{array}$ & 64 & $45,4 *$ & 77 & 54,6 & 141 & \multirow{2}{*}{4,00} & \multirow{2}{*}{0,04} & \multirow{2}{*}{$\begin{array}{c}1,6 \\
(1,009-2,614)\end{array}$} \\
\hline & $\begin{array}{c}\text { No expuesta } \\
(\text { Mamatoco-DJCR) } \\
\mathrm{PM}_{10}<\text { de } 70 \mu \mathrm{g} / \mathrm{m} 3\end{array}$ & 56 & $34,1 *$ & 108 & 65,9 & 164 & & & \\
\hline \multirow{2}{*}{ Fuma } & $\overline{\mathrm{Si}}$ & 24 & 45,3 & 29 & 54,7 & 53 & \multirow{2}{*}{0,94} & \multirow{2}{*}{0,33} & \multirow{2}{*}{ - } \\
\hline & No & 96 & 38,1 & 156 & 61,9 & 252 & & & \\
\hline \multirow{2}{*}{ Hacinamiento } & $\mathrm{Si}$ & 71 & 40,3 & 105 & 59,7 & 176 & \multirow{2}{*}{0,17} & \multirow{2}{*}{0,67} & \multirow{2}{*}{-} \\
\hline & No & 49 & 38,0 & 80 & 62,0 & 129 & & & \\
\hline \multirow{2}{*}{$\begin{array}{l}\text { Techo de } \\
\text { asbesto }\end{array}$} & $\mathrm{Si}$ & 86 & 40,6 & 126 & 59,4 & 212 & \multirow{2}{*}{0,43} & \multirow{2}{*}{0,50} & \multirow{2}{*}{-} \\
\hline & No & 34 & 36,6 & 59 & 63,4 & 93 & & & \\
\hline \multirow{2}{*}{ Piso de tierra } & $\mathrm{Si}$ & 9 & 40,9 & 13 & 59,1 & 22 & \multirow{2}{*}{0,02} & \multirow{2}{*}{0,87} & 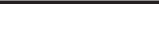 \\
\hline & No & 111 & 39,2 & 172 & 60,8 & 283 & & & \\
\hline Gatos & $\mathrm{Si}$ & 34 & 47,9 & 37 & 52,1 & 71 & 283 & DOP & 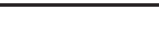 \\
\hline Uatos & No & 86 & 36,8 & 148 & 63,2 & 234 & $2,0 J$ & 0,09 & - \\
\hline Perres & $\mathrm{Si}$ & 34 & 30,1 & 79 & 69,9 & 113 & 644 & 001 & 0,53 \\
\hline rentos & No & 86 & 44,8 & 106 & 55,2 & 192 & 0,44 & 0,01 & $(0,324-0,868)$ \\
\hline & TOTAL & 120 & $39,3^{p}$ & 185 & 60,7 & 305 & & & \\
\hline
\end{tabular}

Tabla 4

Asociación entre síntomas respiratorios del TRS y zona de exposición y presencia de gatos

\begin{tabular}{|c|c|c|c|c|c|c|c|c|c|c|}
\hline & \multirow[t]{2}{*}{ Variables } & \multicolumn{2}{|c|}{$\begin{array}{c}\text { Con } \\
\text { síntomas }\end{array}$} & \multicolumn{2}{|c|}{$\begin{array}{c}\text { Sin } \\
\text { síntomas }\end{array}$} & \multirow[t]{2}{*}{ Total } & \multirow[t]{2}{*}{$\mathrm{Chi}^{2}$} & \multirow[t]{2}{*}{$\mathrm{p}$} & \multirow{2}{*}{$\begin{array}{c}\text { OR } \\
\text { IC } 95 \%\end{array}$} & \multirow{2}{*}{$\begin{array}{l}\text { OR (ajusta- } \\
\text { do) } \\
\text { IC } 95 \%\end{array}$} \\
\hline & & $\mathrm{n}$ & $(\%)$ & $\mathrm{n}$ & $(\%)$ & & & & & \\
\hline \multirow{2}{*}{ Zona } & $\begin{array}{c}\text { Expuesta } \\
\text { (Pescaito-Gaira) } \\
\mathrm{PM}_{10}>\text { de } 70 \mu \mathrm{g} / \mathrm{m} 3\end{array}$ & 63 & 44,7 & 78 & 55,3 & 141 & \multirow{2}{*}{11,3} & \multirow{2}{*}{0,000} & \multirow{2}{*}{$\begin{array}{c}2,27 \\
(1,40-3,67\end{array}$} & \multirow{2}{*}{$\begin{array}{c}2,18 \\
(1,34-3,55)\end{array}$} \\
\hline & $\begin{array}{c}\text { No expuesta } \\
\left(\text { Mamatoco-DJCR) } \mathrm{PM}_{10}<\text { de } 70\right.\end{array}$ & 43 & 26,2 & 121 & 73,8 & 164 & & & & \\
\hline \multirow{2}{*}{ Gatos } & $\mathrm{Si}$ & 33 & 46,5 & 38 & 53,5 & 71 & \multirow{2}{*}{5,61} & \multirow{2}{*}{0,017} & \multirow{2}{*}{$\begin{array}{c}1,91 \\
(1,11-3,29)\end{array}$} & \multirow{2}{*}{$\begin{array}{c}1,7 \\
(1,03-3,11)\end{array}$} \\
\hline & \multirow[t]{2}{*}{ No } & 73 & 31,2 & 161 & 68,8 & 234 & & & & \\
\hline & & 106 & 34,8 & 199 & 65,2 & 305 & & & & \\
\hline
\end{tabular}


Tabla 5

Relación entre los parámetros espirométricos y las zonas expuestas y no expuesta

\begin{tabular}{|c|c|c|c|c|c|c|c|c|c|c|}
\hline \multirow{2}{*}{\multicolumn{2}{|c|}{$\begin{array}{c}\text { Parámetros } \\
\text { espirométricos }\end{array}$}} & \multicolumn{2}{|c|}{ Zonas expuestas } & \multicolumn{2}{|c|}{ Zonas no expuestas } & \multicolumn{2}{|c|}{ Total } & \multirow{2}{*}{$\mathrm{Chi}^{2}$} & \multirow{2}{*}{$\mathrm{p}$} & \multirow{2}{*}{$\begin{array}{c}\text { OR } \\
\mathrm{IC} 95 \%\end{array}$} \\
\hline & & $\bar{n}$ & $(\%)$ & $\bar{n}$ & $(\%)$ & $\mathrm{n}$ & $\mathrm{p}^{*}$ & & & \\
\hline \multirow{2}{*}{ FVC } & $<80 \%$ & 52 & 37,7 & 40 & 26,5 & 92 & \multirow{2}{*}{31,8} & \multirow{2}{*}{4,16} & \multirow{2}{*}{0,04} & \multirow{2}{*}{$\begin{array}{c}1,6 \\
(1,018-2,764)\end{array}$} \\
\hline & $>80 \%$ & 86 & 62,3 & 111 & 73,5 & 197 & & & & \\
\hline \multirow{2}{*}{ FEV 1} & $<80 \%$ & 84 & 60,9 & 38 & 25,2 & 122 & \multirow{2}{*}{42,2} & \multirow{2}{*}{37,6} & \multirow{2}{*}{0,000} & \multirow{2}{*}{$\begin{array}{c}4,62 \\
(2,80-7,64)\end{array}$} \\
\hline & $>80 \%$ & 54 & 39,1 & 113 & 74,8 & 167 & & & & \\
\hline \multirow{2}{*}{ FEV 1/FVC } & $<75 \%$ & 38 & 27,5 & 17 & 11,3 & 55 & \multirow{2}{*}{19} & \multirow{2}{*}{12,3} & \multirow{2}{*}{0,0004} & \multirow{2}{*}{$\begin{array}{c}2,9 \\
(1,598-5,611)\end{array}$} \\
\hline & $>75 \%$ & 100 & 72,5 & 134 & 88,7 & 234 & & & & \\
\hline \multicolumn{2}{|c|}{ Total } & 138 & 47,8 & 151 & 52,2 & 289 & & & & \\
\hline
\end{tabular}

Tabla 6

Asociación entre los patrones espirométricos y las zonas de estudio por exposición a concentraciones de $\mathrm{PM}_{10}$

\begin{tabular}{|c|c|c|c|c|c|c|c|}
\hline \multirow{2}{*}{ Exposición } & $\begin{array}{c}\text { Alteración } \\
\text { Mixta }\end{array}$ & $\begin{array}{c}\text { Obstrucción } \\
\text { Pura }\end{array}$ & $\begin{array}{c}\text { Proceso } \\
\text { restrictivo }\end{array}$ & Normal & Total & $\begin{array}{c}\text { OR } \\
\text { IC 95\% * }\end{array}$ & $\mathrm{p}$ \\
\cline { 2 - 6 } & $\mathrm{n}(\%)$ & $\mathrm{n}(\%)$ & $\mathrm{n}(\%)$ & $\mathrm{n}(\%)$ & & & \\
\hline Zona expuesta & $4(1,4)$ & $34(11,8)$ & $48(16,6)$ & $52(18)$ & 138 & 2,97 & 0,000 \\
\hline Zona no expuesta & $3(1)$ & $14(4,8)$ & $37(12,8)$ & $97(33,6)$ & 151 & $(1,84-4,79)$ & \\
\hline Total & $7(2,4)$ & $48(16,6)$ & $85(29,4)$ & $149(51,6$ & 289 & & \\
\hline
\end{tabular}

*OR $=$ Calculado a partir de datos agrupados como normal y anormal (alteración mixta, obstrucción pura y proceso restrictivo)

(IC 95\% 1,34-3,55)] y tener gatos en la casa, [OR ajustado=1,79 (IC 95\% 1,039$3,11)$ ] fueron factores de riesgo asociados a la presencia de síntomas del TRS.

\section{DISCUSION}

La mayor prevalencia de síntomas del tracto respiratorio superior en relación con los del tracto respiratorio inferior se considera consistente con el hecho de que se midió en relación a los puertos de carbón, que en la ciudad almacenan pero no realizan procesos de combustión que produzcan $\mathrm{PM}_{2,5}$ (partículas finas), las cuales afectan principalmente el tracto respiratorio inferior hasta el nivel alveolar ${ }^{26}$.

La asociación estadísticamente significativa moderada entre los síntomas respi- ratorios y vivir en barrios expuestos a concentraciones de $\mathrm{PM}_{10}$ superiores a la norma concuerda con otros estudios que han encontrado la relación entre $\mathrm{PM}_{10} \mathrm{y}$ síntomas de asma ${ }^{27} \mathrm{y}$ con presencia de $\operatorname{tos}^{28}$.

No se encontró asociación entre la presencia de síntomas respiratorios y las covariables afiliación al sistema de seguridad social, hacinamiento, tabaquismo y contaminantes de la cocina. Al respecto, otros estudios han encontrado que las disparidades socioeconómicas, las condiciones de la vivienda, el acceso a salud y el tabaquismo favorecen la producción de las enfermedades como el asma ${ }^{29}$. Los autores consideran que esto se podría explicar en parte porque la gente prefiere permanecer más tiempo en el exterior de sus viviendas, 
debido a que la temperatura media es de $30^{\circ} \mathrm{C}$ y casi permanentemente hay brisa, lo cual genera que la exposición a contaminantes domiciliarios se reduzca.

Con relación a las mascotas en la vivienda, solamente la presencia de gatos presentó asociación con síntomas del tracto respiratorio superior. Al respecto, algunos autores han encontrado que los alergenos de los gatos impactan en la salud respiratoria hasta antes de los 6 años ${ }^{30} y$ otros han encontrado asociación positiva en niños de 5 a 7 años ${ }^{31}$.

Se evidenció asociación entre la exposición a $\mathrm{PM}_{10}$ y la alteración de la función pulmonar, lo cual concuerda con el estudio sobre función pulmonar realizado en Taiwan, donde encontraron asociación entre $\mathrm{PM}_{10}$ con los patrones respiratorios por espirometría y específicamente con el patrón restrictivo $^{32}$.

Finalmente, las limitaciones de este estudio son las propias del diseño transversal, en el cual la medición de los factores de exposición se restringe a un solo momento en el tiempo. Por lo tanto se considera necesario realizar un estudio longitudinal que permita identificar causalidad entre las concentraciones diarias de $\mathrm{PM}_{10}$ con la presencia de síntomas respiratorios y la alteración en la función pulmonar para identificar el verdadero impacto de la actividad carbonífera sobre la salud infantil en el distrito turístico e histórico de Santa Marta.

Se concluye que en la ciudad de Santa Marta los niños entre 6 y 14 años que viven en zonas expuestas a concentraciones de $\mathrm{PM}_{10}$ superiores a $70 \mu \mathrm{g} / \mathrm{m}^{3}$ tienen mayor riesgo de presentar síntomas de tracto respiratorio superior y alteración de su función pulmonar con relación a aquellos que viven en zonas con concentraciones de $\mathrm{PM}_{10}$ inferiores a $70 \mu \mathrm{g} / \mathrm{m}^{3}$.

\section{BIBLIOGRAFÍA}

1. Schwartz J. Air pollution and daily mortality: A review and meta-analysis. Environ Res J. 1994; 64: 3652.

2. Esplugues A, Ballester F, Estarlich M, Llop S, Fuentes-Leonarte V, Mantilla E, et al. Indoor and outdoor air concentrations of BTEX and determinants in a cohort of one-year old children in Valencia, Spain. Sci Total Environ. 2010; 409 (1): 63-69.

3. Gauderman J, Air Pollution and Children. An Unhealthy Mix (Editorial). N Engl J Med. 2006; 355 (1):78

4. Kim JJ. Ambient air pollution: health hazards to children. Pediatrics. 2004; 114: 1699-1707.

5. Ward D, Ayres I. Particulate air pollution and panel studies in children: a systematic review. Occup Environ Med. 2004; 61(13): 1-12

6. Rowensztein H, Demirdjian G, Rodríguez J. Carga de enfermedad y costos asociados a las internaciones por infección respiratoria aguda en niños. Arch Argent Pediatr. 2007; 105(1): 5-11.

7. Austin J, Selvaraj S, Russell G. Childhood asthma in the highlands of Scotland, morbidity and school absence. Scott Med J. 2004;49: 18-21

8. Castro A, Cunha M, Azevedo G, Silva M, Junger W, De Leon A. Effect of air pollution on lung function in school children in Rio de Janeiro, Brazil. Rev Saude Publica. 2009;43(1): 1-8.

9. Hernández L, Téllez M, Sanín L, Lacasaña M, Campos A, Romieu I. Relación entre consultas a urgencias por enfermedad respiratoria y contaminación atmosférica en Ciudad Juárez, Chihuahua. Salud Publica Mex. 2000;42: 288-297.

10. Barrios S, Peña-Cortés C y Osses-Bustingorry S. Efectos de la contaminación atmosférica por material particulado en las enfermedades respiratorias agudas en menores de 5 años. Cienc Enferm. 2004;10(2): 21 29.

11. Romero-Placeres M, Más-Bermejo P, LacasañaNavarro M, Rojo-Solís M, Téllez M, Aguilar-Valdés J, et al. Contaminación atmosférica, asma bronquial e infecciones respiratorias agudas en menores de edad, de La Habana. Salud Publica Mex. 2004;46(3): 222 233.

12. Aristizabal G, Suescún J, Patiño R. Contaminación del aire y enfermedad respiratoria en la población infantil de Puente Aranda, Santa fe de Bogotá. Universidad del Bosque. Secretaría Distrital de Salud; 1997. 
13. Solarte I, Caicedo M, Restrepo S. Contaminación atmosférica y enfermedad respiratoria en niños menores de 14 años en Santa Fe de Bogotá. Revista Médica Sanitas. 2002; 5: 2-21.

14. Sanhueza P, Vargas C, Mellado P. Impacto de la contaminación del aire por $\mathrm{PM}_{10}$ sobre la mortalidad diaria en Temuco. Rev Med Chile. 2006; 134(6): 754-761.

15. Tellez-Rojo M, Romieu I, Ruiz-Velasco S, Lezana M, Hernandez-Avila M. Daily respiratory mortality and $\mathrm{PM}_{10}$ pollution in Mexico City: importance of considering place of death. Eur Respir J. 2000; 16(3): 391-396

16. Laden F, Schwartz J, Speizer F, Dockery D. Reduction in Fine Particulate Air Pollution and Mortality: Extended Follow-up of the Harvard Six Cities Study. Am J Respir Crit Care Med. 2006; 173: 667-672.

17. García F, Agudelo R, Jiménez K. Distribución espacial y temporal de la concentración de material particulado en Santa Marta, Colombia. Rev Fac Nac Salud Pública. 2006; 24(2): 73-82

18. Manjarrés G, Pinzón G, Linero J. Composición y concentración de material particulado en el aire de un sector del área urbana de Santa Marta (Magdalena, Colombia). Rev Intrópica. 2005; 2: 23-33

19. Mendoza D, Del Castillo L, Lozano S, Jaimes M, Pedrozo J, Abello J. Factores de riesgo asociados con asma alérgica en niños de 4 a 16 años de Santa Marta, Colombia. Rev Duazary. 2008; 5(1): 9-14

20. Organización Mundial de la Salud. Guías de calidad del aire de la OMS relativas al material particulado, el ozono, el dióxido de nitrógeno y el dióxido de azufre. Actualización mundial 2005. Resumen de evaluación de los riesgos. Ginebra: Organización Mundial de la Salud; 2006.

21. Garcia E, Aristizabal G, Vasquez C, Rodriguez-Martinez C, Sarmiento O, Satizabal C.Prevalence of and factors associated with current asthma symptoms in school children aged 6-7 and 13-14 yr old in Bogotá, Colombia. Pediatr Allergy Immunol. 2008;19(4): $307-$ 14.

22. Asher M, Keil U, Anderson H, Beasley R, Crane J, Martinez F, et al. International Study of Asthma and allergies in Childhood (ISAAC): rationale and methods. Eur Respir J. 1995;8: 483-491.

23. Spirometric Classification. ATS. Disponible en: http://www.thoracic.org/clinical/copd-guidelines/forhealth-professionals/definition-diagnosis-and-staging/spirometric-classification.php
24. Miller MR, Hankinson J, Brusasco V, Burgos F, Casaburi R, Coates A, et all. Standardisation of spirometry. Eur Respir J 2005; 26: 319-338. Disponible en: http://www.ersnet.org/

25. Rodríguez L, Rey J, Berena A, Castro H, Niederbacher J, Vera L, et al. Prevalencia de síntomas respiratorios indicativos de asma y asociación con contaminación atmosférica en preescolares de Bucaramanga, Colombia. Biomédica. 2010;30(1): 15-22.

26. Echeverri C, Maya G. Relación entre las partículas finas $(\mathrm{PM} 2.5)$ y respirables $\left(\mathrm{PM}_{10}\right)$ en la ciudad de Medellín. Revista de Ingenierías Universidad de Medellín. 2008;7(12): 23-42.

27. Weinmayr G, Romeo E, De Sario M, Weiland S, Forastiere F. Short-term effects of $\mathrm{PM}_{10}$ and NO2 on respiratory health among children with asthma or asthma-like symptoms: a systematic review and meta-analysis. Environ Health Perspect. 2012; 118 (4): 449-457.

28. Ostro B, Lipsett M, Mann J, Braxton-Owens H, White M. Air pollution and exacerbation of asthma in African-American Children in Los Angeles. Epidemiology. 2001;12(2): 200-208

29. Gold D, Wright R. Population disparities in asthma. Annu Rev Public Health. 2005; 26: 89-113.

30. Mei Chen C, Rzehak P, Zutavern A, Fahlbusch B, Bischof W, Herbarth O, et al. Longitudinal study on cat allergen exposure and the development of allergy in young children. J Allergy Clin Immunol. 2007; 119 (5): 1148-1155

31. Wickman M, Hoek G, Giovannangelo M, Nordling E, Wijga A, de Jongste J, et al. Domestic cat allergen and allergic sensitisation in young children. Int J Hyg Environ Health. 2008; 211(3-4):337-344

32. Chang Y, Wu C, Lee L, Lin R, Yu Y, Chen Y. The short-term effects of air pollution on adolescent lung function in Taiwan. Chemosphere. 2012;97(1): 26-30. 